International Journal of Pure and Applied Mathematics

Volume 94 No. 1 2014, 101-118

ISSN: 1311-8080 (printed version); ISSN: 1314-3395 (on-line version)

url: http://www.ijpam.eu

doi: http://dx.doi.org/10.12732/ijpam.v94i1.11

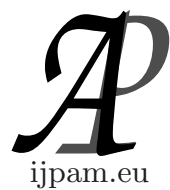

\title{
SOLUTIONS OF FUZZY RELATION EQUATIONS IN GENERALIZED RESIDUATED LATTICES
}

\author{
Yong Chan Kim \\ Department of Mathematics \\ Gangneung-Wonju University \\ Gangneung, Gangwondo, 210-702, KOREA
}

\begin{abstract}
In this paper, we investigate solutions of various types of fuzzy relation equations $A_{i} \circ R=B_{i}, R \circ A_{i}=B_{i}, A_{i} \Rightarrow R=B_{i}$ and $A_{i} \rightarrow R=B_{i}$ in generalized residuated lattice.

We give also some examples.
\end{abstract}

AMS Subject Classification: 03E72, 03G10, 06A15, 06F07

Key Words: fuzzy relation equations, generalized residuated lattices, right $\odot$-preorder, left $\odot$-preorder

\section{Introduction}

Sanchez [14] introduced the theory of fuzzy relation equations with various types of composition: max-min, min-max, min- $\alpha$. Fuzzy relation equations with new types of composition( pseudo t-norm [9], continuous t-norm [15], residuated lattice [11-13]) is developed [8,10]. On the other hand, noncommutative structures play an important role in metric spaces, algebraic structures (groups, rings, quantales, pseudo-BL-algebras) [2-7]. Georgescu and Iorgulescu [5] introduced pseudo MV-algebras as the generalization of the MV-algebras. Georgescu and Popescu [6] introduced generalized residuated lattice as a noncommutative structure.

Received: May 8, 2014

(c) 2014 Academic Publications, Ltd. url: www.acadpubl.eu 
In this paper, we investigate solutions of various types of fuzzy relation equations $A_{i} \circ R=B_{i}, R \circ A_{i}=B_{i}, A_{i} \Rightarrow R=B_{i}$ and $A_{i} \rightarrow R=B_{i}$ in generalized residuated lattice. We give their examples.

\section{Preliminaries}

Definition 2.1. [6] A structure $(L, \vee, \wedge, \odot, \rightarrow, \Rightarrow, \top, \perp)$ is called a generalized residuated lattice if it satisfies the following conditions:

(GR1) $(L, \vee, \wedge, \top, \perp)$ is a bounded lattice where $\top$ is the universal upper bound and $\perp$ denotes the universal lower bound;

$(\operatorname{GR2})(L, \odot, \top)$ is a monoid;

(GR3) it satisfies a residuation, i.e.

$$
a \odot b \leq c \text { iff } a \leq b \rightarrow c \text { iff } b \leq a \Rightarrow c .
$$

Remark 2.2. [6] (1) A generalized residuated lattice is a residuated lattice $(\rightarrow=\Rightarrow)$ iff $\odot$ is commutative.

(2) A left-continuous t-norm $([0,1], \leq, \odot)$ defined by $a \rightarrow b=\bigvee\{c \mid a \odot c \leq$ $b\}$ is a residuated lattice.

(3) Let $(L, \leq, \odot)$ be a quantale. For each $x, y \in L$, we define

$$
\begin{aligned}
& x \rightarrow y=\bigvee\{z \in L \mid z \odot x \leq y\} \\
& x \Rightarrow y=\bigvee\{z \in L \mid x \odot z \leq y\}
\end{aligned}
$$

Then it satisfies Galois correspondence, that is,

$$
(x \odot y) \leq z \text { iff } x \leq(y \rightarrow z) \text { iff } y \leq(x \Rightarrow z) .
$$

Hence $(L, \vee, \wedge, \odot, \rightarrow, \Rightarrow, \top, \perp)$ is a generalized residuated lattice.

(4) A pseudo MV-algebra is a generalized residuated lattice with the law of double negation, that is, $a=\left(a^{*}\right)^{0}=\left(a^{0}\right)^{*}$ where $a^{0}=a \rightarrow \perp$ and $a^{*}=a \Rightarrow \perp$.

In this paper, we assume $(L, \wedge, \vee, \odot, \rightarrow, \Rightarrow, \top, \perp)$ is a generalized residuated lattice. 
Lemma 2.3. [6] For each $x, y, z, x_{i}, y_{i} \in L$, we have the following properties.

(1) If $y \leq z,(x \odot y) \leq(x \odot z), x \rightarrow y \leq x \rightarrow z$ and $z \rightarrow x \leq y \rightarrow x$ for $\rightarrow \in\{\rightarrow, \Rightarrow\}$.

(2) $x \odot y \leq x \wedge y \leq x \vee y$.

(3) $x \rightarrow\left(\bigwedge_{i \in \Gamma} y_{i}\right)=\bigwedge_{i \in \Gamma}\left(x \rightarrow y_{i}\right)$ and $\left(\bigvee_{i \in \Gamma} x_{i}\right) \rightarrow y=\bigwedge_{i \in \Gamma}\left(x_{i} \rightarrow y\right)$ for $\rightarrow \in\{\rightarrow, \Rightarrow\}$.

(4) $x \rightarrow\left(\bigvee_{i \in \Gamma} y_{i}\right) \geq \bigvee_{i \in \Gamma}\left(x \rightarrow y_{i}\right)$, for $\rightarrow \in\{\rightarrow, \Rightarrow\}$.

(5) $\left(\bigwedge_{i \in \Gamma} x_{i}\right) \rightarrow y \geq \bigvee_{i \in \Gamma}\left(x_{i} \rightarrow y\right)$, for $\rightarrow \in\{\rightarrow, \Rightarrow\}$.

(6) $(x \odot y) \rightarrow z=x \rightarrow(y \rightarrow z)$ and $(x \odot y) \Rightarrow z=y \Rightarrow(x \Rightarrow z)$.

(7) $x \rightarrow(y \Rightarrow z)=y \Rightarrow(x \rightarrow z)$ and $x \Rightarrow(y \rightarrow z)=y \rightarrow(x \Rightarrow z)$.

(8) $x \odot(x \Rightarrow y) \leq y$ and $(x \rightarrow y) \odot x \leq y$.

(9) $(x \Rightarrow y) \odot(y \Rightarrow z) \leq x \Rightarrow z$ and $(y \rightarrow z) \odot(x \rightarrow y) \leq x \rightarrow z$.

(10) $(x \Rightarrow z) \leq(y \odot x) \Rightarrow(y \odot z)$ and $(x \rightarrow z) \leq(x \odot y) \rightarrow(z \odot y)$.

(11) $(x \Rightarrow y) \leq(y \Rightarrow z) \rightarrow(x \Rightarrow z)$ and $(x \rightarrow y) \leq(y \rightarrow z) \Rightarrow(x \rightarrow z)$.

(12) $\bigwedge_{i \in \Gamma}\left(x_{i} \rightarrow y_{i}\right) \leq\left(\bigwedge_{i \in \Gamma} x_{i}\right) \rightarrow\left(\bigwedge_{i \in \Gamma} y_{i}\right)$ for $\rightarrow \in\{\rightarrow, \Rightarrow\}$.

(13) $\bigwedge_{i \in \Gamma}\left(x_{i} \rightarrow y_{i}\right) \leq\left(\bigvee_{i \in \Gamma} x_{i}\right) \rightarrow\left(\bigvee_{i \in \Gamma} y_{i}\right)$ for $\rightarrow \in\{\rightarrow, \Rightarrow\}$.

(14) $x \rightarrow y=\top$ iff $x \leq y$ iff $x \Rightarrow y=\top$.

\section{Solutions of Fuzzy Relation Equations in Generalized Residuated} Lattices

Definition 3.1. Let $A_{i} \in L^{U}, R \in L^{U \times V}$ and $B_{i} \in L^{V}$. We define fuzzy relation equations as follows, for each $i \in\{1, \ldots, n\}$,

$$
\begin{aligned}
(1) \quad\left(A_{i} \circ R\right)(v) & =\bigvee_{u \in U}\left(A_{i}(u) \odot R(u, v)\right) \\
& =B_{i}(v), \\
(2)\left(R \circ A_{i}\right)(v) & =\bigvee_{u \in U}\left(R(u, v) \odot A_{i}(u)\right) \\
& =B_{i}(v), \\
\left(\text { 3) }\left(A_{i} \Rightarrow R\right)(v)\right. & =\bigwedge_{v \in V}\left(A_{i}(u) \Rightarrow R(u, v)\right) \\
& =B_{i}(v), \\
\left(\text { 4) }\left(A_{i} \rightarrow R\right)(v)\right. & =\bigwedge_{v \in V}\left(A_{i}(u) \rightarrow R(u, v)\right) \\
& =B_{i}(v) .
\end{aligned}
$$


Let $U=\left\{u_{1}, \ldots, u_{n}\right\}$ be a set, $R \in L^{U \times V}$ an unknown fuzzy relation $A_{1}, \ldots, A_{n} \in L^{U}$ and $B_{1}, \ldots, B_{n} \in L^{V}$. If $v \in V, A_{i}\left(u_{j}\right)=a_{i j}$ for $i, j \in\{1, \ldots, n\}$, $R\left(u_{j}, v\right)=r_{j}, B_{j}(v)=b_{j}$, then the system (1) can be written by

$$
\begin{aligned}
& a_{11} \odot r_{1} \vee \ldots \vee a_{1 n} \odot r_{n}=b_{1} \\
& \text {. } . \text {. } \\
& a_{n 1} \odot r_{1} \vee \ldots \vee a_{n n} \odot r_{n}=b_{n}
\end{aligned}
$$

The system (2) can be written by

$$
\begin{array}{lc}
r_{1} \odot a_{11} \vee \ldots \vee r_{n} \odot a_{1 n} & =b_{1} \\
\cdot \quad \cdot & \cdot \\
: \quad: & : \\
r_{1} \odot a_{n 1} \vee \ldots \vee r_{n} \odot a_{n n} & =b_{n} .
\end{array}
$$

Then system (3) can be written by

$$
\begin{aligned}
& a_{11} \Rightarrow r_{1} \wedge \ldots \wedge a_{1 n} \Rightarrow r_{n} \quad=b_{1} \\
& \cdot \\
& a_{n 1} \Rightarrow r_{1} \wedge \ldots \wedge a_{n n} \Rightarrow r_{n}=b_{n} \text {. }
\end{aligned}
$$

The system (4) can be written by

$$
\begin{array}{lc}
a_{11} \rightarrow r_{1} \wedge \ldots \wedge a_{1 n} \rightarrow r_{n} & =b_{1} \\
\quad . & : \\
: & : \\
a_{n 1} \rightarrow r_{1} \wedge \ldots \wedge a_{n n} \rightarrow r_{n} & =b_{n} .
\end{array}
$$

Theorem 3.2. (1) (5) is solvable iff $r^{\Rightarrow}=\left(r_{1}^{\Rightarrow}, \ldots, r_{n}^{\Rightarrow}\right)$ with $r_{j}^{\Rightarrow}=\bigwedge_{i=1}^{n}\left(a_{i j} \Rightarrow\right.$ $b_{i}$ ) for $j \in\{1, \ldots, n\}$ is the greatest solution.

(2) (6) is solvable iff $r \rightarrow=\left(r_{1} \rightarrow, \ldots, r_{n}\right)$ with $r_{j}=\bigwedge_{i=1}^{n}\left(a_{i j} \rightarrow b_{i}\right)$ for $j \in\{1, \ldots, n\}$ is the greatest solution.

(3) $(7)$ is solvable iff $r^{\odot}=\left(r_{1}^{\odot}, \ldots, r_{n}^{\odot}\right)$ with $r_{k}^{\odot}=\bigvee_{i=1}^{n}\left(a_{i k} \odot b_{i}\right)$ is the least solution. 
(4) (8) is solvable iff $\odot_{r}=\left({ }^{\odot} r_{1}, \ldots,{ }^{\odot} r_{n}\right)$ with ${ }^{\odot} r_{k}=\bigvee_{i=1}^{n}\left(b_{i} \odot a_{i k}\right)$ is the least solution.

Proof. (1) $(\Rightarrow)$ Let $r=\left(r_{1}, \ldots, r_{n}\right)$ be a solution of (5). Since $a_{i k} \odot r_{k} \leq$ $\bigvee_{k=1}^{n}\left(a_{i k} \odot r_{k}\right)=b_{i}, 1 \leq i \leq n$, then $r_{k} \leq a_{i k} \Rightarrow b_{i}$. Hence $r_{k} \leq \bigwedge_{i=1}^{n}\left(a_{i k} \Rightarrow b_{i}\right)$. Put $r_{j}^{\Rightarrow}=\bigwedge_{i=1}^{n}\left(a_{i j} \Rightarrow b_{i}\right)$. So,

$$
\begin{aligned}
b_{i} & =\bigvee_{k=1}^{n}\left(a_{i k} \odot r_{k}\right) \leq \bigvee_{k=1}^{n}\left(a_{i k} \odot \bigwedge_{i=1}^{n}\left(a_{i k} \Rightarrow b_{i}\right)\right) \\
& =\bigvee_{k=1}^{n}\left(a_{i k} \odot r_{k}\right) \leq \bigvee_{j=1}^{n}\left(a_{i j} \odot\left(a_{i j} \Rightarrow b_{i}\right)\right) \leq b_{i} .
\end{aligned}
$$

Thus, $\bigvee_{k=1}^{n}\left(a_{i k} \odot r_{k}\right)=b_{i}$. Hence $r \Rightarrow=\left(r_{1}^{\Rightarrow}, \ldots, r_{n}^{\Rightarrow}\right)$ with $r_{j}^{\Rightarrow}=\bigwedge_{i=1}^{n}\left(a_{i j} \Rightarrow b_{i}\right)$ is the greatest solution.

(2) $(\Rightarrow)$ Let $r=\left(r_{1}, \ldots, r_{n}\right)$ be a solution of (6). Since $r_{k} \odot a_{i k} \leq \bigvee_{k=1}^{n}\left(r_{k} \odot\right.$ $\left.a_{i k}\right)=b_{i}, 1 \leq i \leq n$, then $r_{k} \leq a_{i k} \rightarrow b_{i}$. Hence $r_{k} \leq \bigwedge_{i=1}^{n}\left(a_{i k} \rightarrow b_{i}\right)$. Put $r_{j} \overrightarrow{=} \bigwedge_{i=1}^{n}\left(a_{i j} \rightarrow b_{i}\right)$. So,

$$
\begin{aligned}
b_{i} & =\bigvee_{k=1}^{n}\left(r_{k} \odot a_{i k}\right) \leq \bigvee_{k=1}^{n}\left(\bigwedge_{i=1}^{n}\left(a_{i k} \rightarrow b_{i}\right) \odot a_{i k}\right) \\
& =\bigvee_{k=1}^{n}\left(r_{k} \odot a_{i k}\right) \leq \bigvee_{j=1}^{n}\left(\left(a_{i j} \rightarrow b_{i}\right) \odot a_{i j}\right) \leq b_{i} .
\end{aligned}
$$

Thus, $\bigvee_{k=1}^{n}\left(r_{k} \odot a_{i k}\right)=b_{i}$. Hence $r \rightarrow=\left(r_{1}, \ldots, r_{n}\right)$ with $r_{j}=\bigwedge_{i=1}^{n}\left(a_{i j} \rightarrow b_{i}\right)$ is the greatest solution.

(3) Let $r=\left(r_{1}, \ldots, r_{n}\right)$ be a solution of (7). Since $b_{i}=\bigwedge_{k=1}^{n}\left(a_{i k} \Rightarrow r_{k}\right) \leq$ $a_{i k} \Rightarrow r_{k}$, then $r_{k} \geq a_{i k} \odot b_{i}$. Thus $r_{k} \geq \bigvee_{i=1}^{n}\left(a_{i k} \odot b_{i}\right)$. Put $r_{k}^{\odot}=\bigvee_{i=1}^{n}\left(a_{i k} \odot b_{i}\right)$.

$$
\begin{aligned}
b_{i} & \left.\leq \bigwedge_{k=1}^{n}\left(a_{i k} \Rightarrow a_{i k} \odot b_{i}\right)\right) \\
& \leq \bigwedge_{k=1}^{n}\left(a_{i k} \Rightarrow \bigvee_{p=1}^{m}\left(a_{p k} \odot b_{p}\right)\right) \\
& =\bigwedge_{k=1}^{n}\left(a_{i k} \Rightarrow r_{k}^{\odot}\right) \\
& \leq \bigwedge_{k=1}^{n}\left(a_{i k} \Rightarrow r_{k}\right)=b_{i} .
\end{aligned}
$$

Thus, $\bigwedge_{k=1}^{n}\left(a_{i k} \Rightarrow r_{k}^{\odot}\right)=b_{i}$. Hence $r^{\odot}=\left(r_{1}^{\odot}, \ldots, r_{n}^{\odot}\right)$ with $r_{k}^{\odot}=\bigvee_{k}^{n}\left(a_{i k} \odot b_{i}\right)$ is the least solution.

(4) Let $r=\left(r_{1}, \ldots, r_{n}\right)$ be a solution of (8). Since $b_{i}=\bigwedge_{k=1}^{n}\left(a_{i k} \rightarrow r_{k}\right) \leq$ $a_{i k} \rightarrow r_{k}$, then $r_{k} \geq b_{i} \odot a_{i k}$. Thus $r_{k} \geq \bigvee_{i=1}^{n}\left(b_{i} \odot a_{i k}\right)$.

$$
\begin{aligned}
b_{i} & \leq \bigwedge_{k=1}^{n}\left(a_{i k} \rightarrow\left(b_{i} \odot a_{i k}\right)\right) \\
& \leq \bigwedge_{k=1}^{n}\left(a_{i k} \rightarrow \bigvee_{p=1}^{n}\left(b_{p} \odot a_{p k}\right)\right) \\
& =\bigwedge_{k=1}^{n}\left(a_{i k} \rightarrow \odot r_{k}\right) \\
& \leq \bigwedge_{k=1}^{n}\left(a_{i k} \rightarrow r_{k}\right)=b_{i} .
\end{aligned}
$$

Thus, $\bigwedge_{k=1}^{n}\left(a_{i k} \rightarrow{ }^{\odot} r_{k}\right)=b_{i}$. Hence $\odot^{\odot} r=\left({ }^{\odot} r_{1}, \ldots,{ }^{\odot} r_{n}\right)$ with $\odot r_{k}=$ $\bigvee_{i=1}^{n}\left(b_{i} \odot a_{i k}\right)$ is the least solution. 
Theorem 3.3. (1) If (5) is solvable, then $\bigwedge_{k=1}^{n}\left(a_{i k} \rightarrow a_{j k}\right) \leq b_{i} \rightarrow b_{j}$.

(2) If (6) is solvable, then $\bigwedge_{k=1}^{n}\left(a_{i k} \Rightarrow a_{j k}\right) \leq b_{i} \Rightarrow b_{j}$.

(3) If (7) is solvable, then $\bigwedge_{k=1}^{n}\left(a_{j k} \Rightarrow a_{i k}\right) \leq b_{i} \rightarrow b_{j}$.

(4) If (8) is solvable, then $\bigwedge_{k=1}^{n}\left(a_{j k} \rightarrow a_{i k}\right) \leq b_{i} \Rightarrow b_{j}$.

Proof. (1) Let $r=\left(r_{1}, \ldots, r_{n}\right)$ be a solution of (5). Since $\bigvee_{k=1}^{n}\left(a_{i k} \odot r_{k}\right)=b_{i}$, by Lemma $2.3(10,13)$, we have

$$
\begin{aligned}
b_{i} \rightarrow b_{j} & =\bigvee_{k=1}^{n}\left(a_{i k} \odot r_{k}\right) \rightarrow \bigvee_{k=1}^{n}\left(a_{j k} \odot r_{k}\right) \\
& \geq \bigwedge_{k=1}^{n}\left(\left(a_{i k} \odot r_{k}\right) \rightarrow\left(a_{j k} \odot r_{k}\right)\right) \\
& \geq \bigwedge_{k=1}^{n}\left(a_{i k} \rightarrow a_{j k}\right) .
\end{aligned}
$$

(2) Let $r=\left(r_{1}, \ldots, r_{n}\right)$ be a solution of $(6)$. Since $\bigvee_{k=1}^{n}\left(r_{k} \odot a_{i k}\right)=b_{i}$, by Lemma $2.3(10,13)$, we have

$$
\begin{aligned}
b_{i} \Rightarrow b_{j} & =\bigvee_{k=1}^{n}\left(r_{k} \odot a_{i k}\right) \Rightarrow \bigvee_{k=1}^{n}\left(r_{k} \odot a_{j k}\right) \\
& \geq \bigwedge_{k=1}^{n}\left(\left(r_{k} \odot a_{i k}\right) \Rightarrow\left(r_{k} \odot a_{j k}\right)\right) \\
& \geq \bigwedge_{k=1}^{n}\left(a_{i k} \Rightarrow a_{j k}\right) .
\end{aligned}
$$

(3) Let $r=\left(r_{1}, \ldots, r_{n}\right)$ be a solution of (7). Since $\bigwedge_{k=1}^{n}\left(a_{i k} \Rightarrow r_{k}\right)=b_{i}$, by Lemma $2.3(11,12)$, we have

$$
\begin{aligned}
b_{i} \rightarrow b_{j} & =\bigwedge_{k=1}^{n}\left(a_{i k} \Rightarrow r_{k}\right) \rightarrow \bigwedge_{k=1}^{n}\left(a_{j k} \Rightarrow r_{k}\right) \\
& \geq \bigwedge_{k=1}^{n}\left(\left(a_{i k} \Rightarrow r_{k}\right) \rightarrow\left(a_{j k} \Rightarrow r_{k}\right)\right) \\
& \geq \bigwedge_{k=1}^{n}\left(a_{j k} \Rightarrow a_{i k}\right) .
\end{aligned}
$$

(4) Let $r=\left(r_{1}, \ldots, r_{n}\right)$ be a solution of (8). Since $\bigwedge_{k=1}^{n}\left(a_{j k} \rightarrow r_{k}\right)=b_{j}$, by Lemma $2.3(11,12)$, we have

$$
\begin{aligned}
b_{i} \Rightarrow b_{j} & =\bigwedge_{k=1}^{n}\left(a_{i k} \rightarrow r_{k}\right) \Rightarrow \bigwedge_{k=1}^{n}\left(a_{j k} \rightarrow r_{k}\right) \\
& \geq \bigwedge_{k=1}^{n}\left(\left(a_{i k} \rightarrow r_{k}\right) \Rightarrow\left(a_{j k} \rightarrow r_{k}\right)\right) \\
& \geq \bigwedge_{k=1}^{n}\left(a_{j k} \rightarrow a_{i k}\right) .
\end{aligned}
$$

Definition 3.4.Let $U$ be a set. A function $P: U \times U \rightarrow L$ is called a right $\odot$-preorder on $U$ if it satisfies the following conditions:

(R) (reflexive) $P(u, u)=\top$ for all $u \in U$,

(RT) (right transitive) $P(u, v) \odot P(v, w) \leq P(u, w)$, for all $u, v, w \in U$.

A function $P: U \times U \rightarrow L$ is called a left $\odot$-preorder on $U$ if it satisfies $(\mathrm{R})$ and the following condition: 
(LT) (left transitive) $P(v, w) \odot P(u, v) \leq P(u, w)$, for all $u, v, w \in U$.

Remark 3.5. (1) If $P: U \times U \rightarrow L$ is a right (resp. left) $\odot$-preorder and define $P^{t}(u, v)=P(v, u)$, then $P^{t}: U \times U \rightarrow L$ is a left (resp. right) $\odot$-preorder.

(2) For $P \in L^{U \times U}$, we define $(P \circ P)(u, w)=\bigvee_{v \in U}(P(u, v) \odot P(v, w))$. Then $P$ is right transitive iff $P \circ P \leq P$. In particular, $P$ is left transitive iff $P^{t} \circ P^{t} \leq P^{t}$.

(3) Let $U$ be a set. Define $P_{1}, P_{2}: L^{U} \times L^{U} \rightarrow L$ as follows:

$$
\begin{aligned}
& P_{1}(A, B)=\bigwedge_{u \in U}(A(u) \Rightarrow B(u)), \\
& P_{2}(A, B)=\bigwedge_{u \in U}(A(u) \rightarrow B(u)) .
\end{aligned}
$$

By Lemma 2.3(9), $P_{1}$ is a right $\odot$-preorder on $L^{U}$ and $P_{2}$ is a left $\odot$-preorder on $L^{U}$.

A fuzzy set $A \in L^{X}$ is call normal if there exists $u \in U$ such that $A(u)=\top$.

Theorem 3.6. Let $U=\left\{u_{1}, \ldots, u_{n}\right\}$ be a set and $A_{i} \in L^{U}$ normal for all $i \in\{1, \ldots, n\}$ such that $A_{i}\left(u_{i}\right)=\top$. Then the following statements are equivalent.

(1) There exists a right $\odot$-preorder $P$ such that $A_{i}(u)=P\left(u_{i}, u\right)$ for all $i \in\{1, \ldots, n\}, u \in U$.

(2) $A_{i}(u)=\bigwedge_{k=1}^{n}\left(A_{k}\left(u_{i}\right) \Rightarrow A_{k}(u)\right)$ for all $i \in\{1, \ldots, n\}$.

(3) $A_{i}\left(u_{j}\right)=\bigwedge_{u \in U}\left(A_{j}(u) \rightarrow A_{i}(u)\right)$ for all $i, j \in\{1, \ldots, n\}$.

Proof. $\quad(1) \Rightarrow(2)$. Since $P\left(u_{i}, u\right) \odot P(u, v) \leq P\left(u_{i}, v\right)$, then $P(u, v) \leq$ $P\left(u_{i}, u\right) \Rightarrow P\left(u_{i}, v\right)=A_{i}(u) \Rightarrow A_{i}(v)$. Hence $P(u, v) \leq \bigwedge_{k=1}^{n}\left(A_{k}(u) \Rightarrow A_{k}(v)\right)$. So, $A_{i}(u)=P\left(u_{i}, u\right) \leq \bigwedge_{k=1}^{n}\left(A_{k}\left(u_{i}\right) \Rightarrow A_{k}(u)\right) \leq A_{i}\left(u_{i}\right) \Rightarrow A_{i}(u)=A_{i}(u)$. Thus $A_{i}(u)=\bigwedge_{k=1}^{n}\left(A_{k}\left(u_{i}\right) \Rightarrow A_{k}(u)\right)$ for all $i \in\{1, \ldots, n\}, u \in U$.

$(2) \Rightarrow(3)$. Since

$$
\begin{aligned}
& \bigwedge_{k=1}^{n}\left(A_{k}\left(u_{i}\right) \Rightarrow A_{k}\left(u_{j}\right)\right) \odot \bigwedge_{k=1}^{n}\left(A_{k}\left(u_{j}\right) \Rightarrow A_{k}(u)\right) \\
& \leq \bigwedge_{k=1}^{n}\left(A_{k}\left(u_{i}\right) \Rightarrow A_{k}(u)\right) \\
& \text { iff } A_{i}\left(u_{j}\right) \odot A_{j}(u) \leq A_{i}(u),
\end{aligned}
$$

then $A_{i}\left(u_{j}\right) \leq \bigwedge_{u \in U}\left(A_{j}(u) \rightarrow A_{i}(u)\right) \leq A_{j}\left(u_{j}\right) \rightarrow A_{i}\left(u_{j}\right)=A_{i}\left(u_{j}\right)$ for all $i, j \in\{1, \ldots, n\}$. Thus, $A_{i}\left(u_{j}\right)=\bigwedge_{u \in U}\left(A_{j}(u) \rightarrow A_{i}(u)\right)$.

$(3) \Rightarrow(1)$. Put $P(u, v)=\bigwedge_{k=1}^{n}\left(A_{k}(u) \Rightarrow A_{k}(v)\right)$. Then $P$ is a right $\odot-$ preorder. Thus, $P\left(u_{i}, u\right)=\bigwedge_{k=1}^{n}\left(A_{k}\left(u_{i}\right) \Rightarrow A_{k}(u)\right) \leq A_{i}\left(u_{i}\right) \Rightarrow A_{i}(u)=A_{i}(u)$. 
Since $A_{i}\left(u_{j}\right)=\bigwedge_{x \in X}\left(A_{j}(u) \rightarrow A_{i}(u)\right) \leq A_{j}(u) \rightarrow A_{i}(u)$, then $A_{j}(u) \leq$ $A_{i}\left(u_{j}\right) \Rightarrow A_{i}(u)$. Exchange $i$ and $j$. Then $A_{i}(u) \leq A_{j}\left(u_{i}\right) \Rightarrow A_{j}(u)$. So, $A_{i}(u) \leq \bigwedge_{k=1}^{n}\left(A_{k}\left(u_{i}\right) \Rightarrow A_{k}(u)\right)$. Hence $A_{i}(u)=P\left(u_{i}, u\right)$.

Theorem 3.7. Let $U=\left\{u_{1}, \ldots, u_{n}\right\}$ be a set and $A_{i} \in L^{U}$ normal for $i \in$ $\{1, \ldots, n\}$ such that $A_{i}\left(u_{i}\right)=\top$. Then the following statements are equivalent.

(1) There exists a left $\odot$-preorder $P$ such that $A_{i}(u)=P\left(u_{i}, u\right)$ for all $i \in\{1, \ldots, n\}, u \in U$.

(2) $A_{i}(u)=\bigwedge_{k=1}^{n}\left(A_{k}\left(u_{i}\right) \rightarrow A_{k}(u)\right)$ for all $i \in\{1, \ldots, n\}, u \in U$.

(3) $A_{i}\left(u_{j}\right)=\bigwedge_{u \in U}\left(A_{j}(u) \Rightarrow A_{i}(u)\right)$ for all $i, j \in\{1, \ldots, n\}$.

Proof. $(1) \Rightarrow(2)$. Since $P$ is a left $\odot$-preorder, then $P(u, v) \odot P\left(u_{i}, u\right) \leq$ $P\left(u_{i}, v\right)$. Thus, $P(u, v) \leq P\left(u_{i}, u\right) \rightarrow P\left(u_{i}, v\right)=A_{i}(u) \rightarrow A_{i}(v)$. Hence $P(u, v) \leq \bigwedge_{k=1}^{n}\left(A_{k}(u) \rightarrow A_{k}(v)\right)$. So, $A_{i}(u)=P\left(u_{i}, u\right) \leq \bigwedge_{k=1}^{n}\left(A_{k}\left(u_{i}\right) \rightarrow\right.$ $\left.A_{k}(u)\right) \leq A_{i}\left(u_{i}\right) \rightarrow A_{i}(u)=A_{i}(u)$. Thus $A_{i}(u)=\bigwedge_{k=1}^{n}\left(A_{k}\left(u_{i}\right) \rightarrow A_{k}(u)\right)$ for all $i \in\{1, \ldots, n\}, u \in U$.

$(2) \Rightarrow(3)$. Since

$$
\begin{aligned}
& \bigwedge_{k=1}^{n}\left(A_{k}\left(u_{j}\right) \rightarrow A_{k}(u)\right) \odot \bigwedge_{k=1}^{n}\left(A_{k}\left(u_{i}\right) \rightarrow A_{k}\left(u_{j}\right)\right) \\
& \leq \bigwedge_{k=1}^{n}\left(A_{k}\left(u_{i}\right) \rightarrow A_{k}(u)\right) \\
& \operatorname{iff} A_{j}(u) \odot A_{i}\left(u_{j}\right) \leq A_{i}(u),
\end{aligned}
$$

then $A_{i}\left(u_{j}\right) \leq \bigwedge_{u \in U}\left(A_{j}(u) \Rightarrow A_{i}(u)\right) \leq A_{j}\left(u_{j}\right) \Rightarrow A_{i}\left(u_{j}\right)=A_{i}\left(u_{j}\right)$ for all $i, j \in\{1, \ldots, n\}$. Thus, $A_{i}\left(u_{j}\right)=\bigwedge_{u \in U}\left(A_{j}(u) \Rightarrow A_{i}(u)\right)$.

$(3) \Rightarrow(1)$. Put $P(u, v)=\bigwedge_{k=1}^{n}\left(A_{k}(u) \rightarrow A_{k}(v)\right)$. Then $P$ is a left $\odot-$ preorder. So, $P\left(u_{i}, u\right)=\bigwedge_{k=1}^{n}\left(A_{k}\left(u_{i}\right) \rightarrow A_{k}(u)\right) \leq A_{i}\left(u_{i}\right) \rightarrow A_{i}(u)=A_{i}(u)$.

Since $A_{i}\left(u_{j}\right)=\bigwedge_{u \in U}\left(A_{j}(u) \Rightarrow A_{i}(u)\right) \leq A_{j}(u) \Rightarrow A_{i}(u)$, then $A_{j}(u) \leq$ $A_{i}\left(u_{j}\right) \rightarrow A_{i}(u)$. Exchange $i$ and $j$. Then $A_{i}(u) \leq \bigwedge_{k=1}^{n}\left(A_{k}\left(u_{i}\right) \rightarrow A_{k}(u)\right.$. Hence $A_{i}(u)=P\left(u_{i}, u\right)$.

From Theorems 3.6 and 3.7, we obtain the following corollary.

Corollary 3.8. Let $U=\left\{u_{1}, \ldots, u_{n}\right\}$ be a set. Then the following statements hold.

(1) If $P \in L^{U \times U}$ is a right $\odot$-preorder and we put $A_{i}(u)=P\left(u_{i}, u\right) \in L^{U}$, then $A_{i}\left(u_{i}\right)=a_{i i}=\top$ and for all $i \in\{1, \ldots, n\}$,

$$
A_{i}\left(u_{j}\right)=a_{i j}=\bigwedge_{k=1}^{n}\left(a_{k i} \Rightarrow a_{k j}\right)=\bigwedge_{k=1}^{n}\left(a_{j k} \rightarrow a_{i k}\right)
$$


(2) If $P \in L^{U \times U}$ is a left $\odot$-preorder and we put $A_{i}(u)=P\left(u_{i}, u\right) \in L^{U}$, then $A_{i}\left(u_{i}\right)=a_{i i}=\top$ and for all $i \in\{1, \ldots, n\}$,

$$
A_{i}\left(u_{j}\right)=a_{i j}=\bigwedge_{k=1}^{n}\left(a_{k i} \rightarrow a_{k j}\right)=\bigwedge_{k=1}^{n}\left(a_{j k} \Rightarrow a_{i k}\right) .
$$

Theorem 3.9. Let $A_{i} \in L^{U}$ be normal for $1 \leq i \leq n$ such that $a_{i i}=\top$ and $a_{i j} \leq \bigwedge_{k=1}^{n}\left(a_{j k} \rightarrow a_{i k}\right)$ for all $i, j \in\{1, \ldots, n\}$. Then the following statements hold.

(1) $\bigvee_{j=1}^{n}\left(a_{i j} \odot r_{j}\right)=b_{i}$ is solvable iff $a_{i j} \leq b_{j} \rightarrow b_{i}$ for all $i, j \in\{1, \ldots, n\}$.

(2) $\bigwedge_{j=1}^{n}\left(a_{i j} \rightarrow r_{j}\right)=b_{i}$ is solvable iff $a_{i j} \leq b_{i} \Rightarrow b_{j}$ for all $i, j \in\{1, \ldots, n\}$.

Proof. (1) $(\Rightarrow)$ Let $\bigvee_{j=1}^{n}\left(a_{i j} \odot r_{j}\right)=b_{i}$ be solvable. Then $r_{j}=\bigwedge_{k=1}^{n}\left(a_{k j} \Rightarrow\right.$ $\left.b_{k}\right)$ is a solution. So, $\bigvee_{j=1}^{n}\left(a_{i j} \odot r_{j}\right)=b_{i}$. Since $a_{i j} \leq a_{j k} \rightarrow a_{i k}$ iff $a_{j k} \leq a_{i j} \Rightarrow$ $a_{i k}$, then

$$
\bigvee_{k=1}^{n}\left(\left(a_{i j} \Rightarrow a_{i k}\right) \odot r_{k}\right) \geq \bigvee_{k=1}^{n}\left(a_{j k} \odot r_{k}\right)=b_{j}
$$

Since $r_{k} \Rightarrow a_{i k} \Rightarrow b_{i}$,

$$
\begin{aligned}
a_{i j} \Rightarrow b_{i} & \geq \bigvee_{k=1}^{n}\left(\left(a_{i j} \Rightarrow a_{i k}\right) \odot\left(a_{i k} \Rightarrow b_{i}\right)\right) \\
& \geq \bigvee_{k=1}^{n}\left(\left(a_{i j} \Rightarrow a_{i k}\right) \odot r_{k}^{\Rightarrow}\right) \geq b_{j} .
\end{aligned}
$$

Since $a_{i j} \Rightarrow b_{i} \geq b_{j}$ iff $a_{i j} \leq b_{j} \rightarrow b_{i}$, then $a_{i j} \leq b_{j} \rightarrow b_{i}$.

$(\Leftarrow) r_{j} \Rightarrow \bigwedge_{k=1}^{n}\left(a_{k j} \Rightarrow b_{k}\right)$ is a solution from the following statement:

$$
\begin{aligned}
& \bigvee_{j=1}^{n}\left(a_{i j} \odot r_{j}\right) \leq \bigvee_{j=1}^{n}\left(a_{i j} \odot\left(a_{i j} \Rightarrow b_{i}\right)\right) \leq b_{i}, \\
& \quad \bigvee_{j=1}^{n}\left(a_{i j} \odot r_{j}\right) \geq a_{i i} \odot r_{i} \Rightarrow \top \odot r_{i} \\
& \quad=\bigwedge_{j=1}^{n}\left(a_{j i} \Rightarrow b_{j}\right) \geq b_{i}
\end{aligned}
$$

because $a_{j i} \leq b_{i} \rightarrow b_{j}$ iff $b_{i} \leq a_{j i} \Rightarrow b_{j}$.

$(2)(\Rightarrow)$ Let $\bigwedge_{j=1}^{n}\left(a_{i j} \rightarrow r_{j}\right)=b_{i}$ be solvable. Then ${ }^{{ }} r_{k}=\bigvee_{j=1}^{n}\left(b_{j} \odot a_{j k}\right)$ is a solution. That is, $\bigwedge_{k=1}^{n}\left(a_{i k} \rightarrow^{\odot} r_{k}\right)=b_{i}$. Thus

$$
\bigwedge_{k=1}^{n}\left(a_{j k} \rightarrow b_{i} \odot a_{i k}\right) \leq b_{j} .
$$

Since $a_{i j} \leq a_{j k} \rightarrow a_{i k}$,

$$
b_{i} \odot a_{i j} \odot a_{j k} \leq b_{i} \odot\left(a_{j k} \rightarrow a_{i k}\right) \odot a_{j k} \leq b_{i} \odot a_{i k}
$$


Thus $b_{i} \odot a_{i j} \leq a_{j k} \rightarrow b_{i} \odot a_{i k}$. So, $b_{i} \odot a_{i j} \leq \bigwedge_{k=1}^{n}\left(a_{j k} \rightarrow b_{i} \odot a_{i k}\right) \leq b_{j}$. Thus, $a_{i j} \leq b_{i} \Rightarrow b_{j}$.

$(\Leftarrow)$ We will show that $\odot_{r_{k}}=\bigvee_{j=1}^{n}\left(b_{j} \odot a_{j k}\right)$ is a solution.

$$
\bigwedge_{k=1}^{n}\left(a_{i k} \rightarrow{ }^{\odot} r_{k}\right) \geq \bigwedge_{k=1}^{n}\left(a_{i k} \rightarrow b_{i} \odot a_{i k}\right) \geq b_{i} .
$$

On the other hand, since $a_{i j} \leq b_{i} \Rightarrow b_{j}$, we have

$$
\begin{aligned}
& \bigwedge_{k=1}^{n}\left(a_{i k} \rightarrow \odot r_{k}\right) \leq a_{i i} \rightarrow \odot r_{i}=\top \rightarrow \odot r_{i}=\odot r_{i} \\
& =\bigvee_{j=1}^{n}\left(b_{j} \odot a_{j i}\right) \leq \bigvee_{j=1}^{n}\left(b_{j} \odot\left(b_{j} \Rightarrow b_{i}\right)\right) \leq b_{i} .
\end{aligned}
$$

From Theorem 3.9, we obtain the following corollary.

Corollary 3.10. Let $U=\left\{u_{1}, \ldots, u_{n}\right\}$ be a set and $P \in L^{U \times U}$ a right $\odot$-preorder with $A_{i}(u)=P\left(u_{i}, u\right) \in L^{U}$. Then the following statements hold.

(1) $\bigvee_{j=1}^{n}\left(a_{i j} \odot r_{j}\right)=b_{i}$ is solvable iff $a_{i j}=\bigwedge_{k=1}^{n}\left(a_{k i} \Rightarrow a_{k j}\right)=\bigwedge_{k=1}^{n}\left(a_{j k} \rightarrow\right.$ $\left.a_{i k}\right) \leq b_{j} \rightarrow b_{i}$ for all $i, j \in\{1, \ldots, n\}$.

(2) $\bigwedge_{j=1}^{n}\left(a_{i j} \rightarrow r_{j}\right)=b_{i}$ is solvable iff $a_{i j}=\bigwedge_{k=1}^{n}\left(a_{k i} \Rightarrow a_{k j}\right)=\bigwedge_{k=1}^{n}\left(a_{j k} \rightarrow\right.$ $\left.a_{i k}\right) \leq b_{i} \Rightarrow b_{j}$ for all $i, j \in\{1, \ldots, n\}$.

Theorem 3.11. Let $A_{i} \in L^{U}$ be normal for $1 \leq i \leq n$ such that $a_{i i}=\top$ and $a_{i j} \leq \bigwedge_{k=1}^{n}\left(a_{j k} \Rightarrow a_{i k}\right)$ for all $i, j \in I$. Then the following statements hold.

(1) $\bigvee_{j=1}^{n}\left(r_{j} \odot a_{i j}\right)=b_{i}$ is solvable iff $a_{i j} \leq b_{j} \Rightarrow b_{i}$ for all $i, j=\{1, \ldots, n\}$.

(2) $\bigwedge_{j=1}^{n}\left(a_{i j} \Rightarrow r_{j}\right)=b_{i}$ is solvable iff $a_{i j} \leq b_{i} \rightarrow b_{j}$ for all $i, j=\{1, \ldots, n\}$.

Proof. $(1)(\Rightarrow)$ Let $\bigvee_{j=1}^{n}\left(r_{j} \odot a_{i j}\right)=b_{i}$ be solvable. Then $r_{j}=\bigwedge_{k=1}^{n}\left(a_{k j} \rightarrow\right.$ $\left.b_{k}\right)$ is a solution. So, $\bigvee_{j=1}^{n}\left(r_{j} \odot a_{i j}\right)=b_{i}$. Since $a_{i j} \leq a_{j k} \Rightarrow a_{i k}$ iff $a_{j k} \leq a_{i j} \rightarrow$ $a_{i k}$, then

$$
\bigvee_{k=1}^{n}\left(r_{k} \odot\left(a_{i j} \rightarrow a_{i k}\right)\right) \geq \bigvee_{k=1}^{n}\left(r_{k} \odot a_{j k}\right)=b_{j}
$$

Since $r_{k} \leq a_{i k} \rightarrow b_{i}$

$$
\begin{aligned}
a_{i j} \rightarrow b_{i} & \geq \bigvee_{k=1}^{n}\left(\left(a_{i k} \rightarrow b_{i}\right) \odot\left(a_{i j} \rightarrow a_{i k}\right)\right. \\
& \geq \bigvee_{k=1}^{n}\left(r_{k} \odot\left(a_{i j} \rightarrow a_{i k}\right)\right) \geq b_{j}
\end{aligned}
$$

Since $a_{i j} \rightarrow b_{i} \geq b_{j}$ iff $a_{i j} \leq b_{j} \Rightarrow b_{i}$, then $a_{i j} \leq b_{j} \Rightarrow b_{i}$.

$(\Leftarrow) r_{j}=\bigwedge_{k=1}^{n}\left(a_{k j} \rightarrow b_{k}\right)$ is a solution from the following statement:

$$
\bigvee_{j=1}^{n}\left(r_{j} \odot a_{i j}\right) \leq \bigvee_{j=1}^{n}\left(\left(a_{i j} \rightarrow b_{i}\right) \odot a_{i j}\right) \leq b_{i},
$$




$$
\begin{aligned}
\bigvee_{j=1}^{n}\left(r_{j} \odot a_{i j}\right) & \geq r_{i} \odot a_{i i}=r_{i} \odot \top \\
& =\bigwedge_{k=1}^{n}\left(a_{k i} \rightarrow b_{k}\right) \geq b_{i}
\end{aligned}
$$

because $a_{j i} \leq b_{i} \Rightarrow b_{j}$ iff $b_{i} \leq a_{j i} \rightarrow b_{j}$.

$(2)(\Rightarrow)$ Let $\bigwedge_{k=1}^{n}\left(a_{i k} \Rightarrow r_{k}\right)=b_{i}$ be solvable. Then $r_{k}^{\odot}=\bigvee_{j=1}^{n}\left(a_{j k} \odot b_{j}\right)$ is a solution. That is, $\bigwedge_{k=1}^{n}\left(a_{i k} \Rightarrow r_{k}^{\odot}\right)=b_{i}$. Thus

$$
\bigwedge_{k=1}^{n}\left(a_{j k} \Rightarrow a_{i k} \odot b_{i}\right) \leq b_{j}
$$

Since $a_{i j} \leq a_{j k} \Rightarrow a_{i k}$,

$$
a_{j k} \odot a_{i j} \odot b_{i} \leq a_{j k} \odot\left(a_{j k} \Rightarrow a_{i k}\right) \odot b_{i} \leq a_{i k} \odot b_{i}
$$

Thus $a_{i j} \odot b_{i} \leq a_{j k} \Rightarrow a_{i k} \odot b_{i}$. So, $a_{i j} \odot b_{i} \leq \bigwedge_{k=1}^{n}\left(a_{j k} \Rightarrow a_{i k} \odot b_{i}\right) \leq b_{j}$.

$(\Leftarrow)$ We will show that ${ }^{\odot} r_{k}=\bigvee_{j=1}^{n}\left(b_{j} \odot a_{j k}\right)$ is a solution.

$$
\bigwedge_{k=1}^{n}\left(a_{i k} \rightarrow \odot r_{k}\right) \geq \bigwedge_{k=1}^{n}\left(a_{i k} \rightarrow b_{i} \odot a_{i k}\right) \geq b_{i} .
$$

On the other hand, since $a_{i j} \leq b_{i} \Rightarrow b_{j}$, we have

$$
\begin{aligned}
& \bigwedge_{k=1}^{n}\left(a_{i k} \rightarrow \odot r_{k}\right) \leq a_{i i} \rightarrow \odot r_{i}=\top \rightarrow \odot r_{i}=\odot r_{i} \\
& =\bigvee_{j=1}^{n}\left(b_{j} \odot a_{j i}\right) \leq \bigvee_{j=1}^{n}\left(b_{j} \odot\left(b_{j} \Rightarrow b_{i}\right)\right) \leq b_{i} .
\end{aligned}
$$

Thus, $\bigwedge_{k=1}^{n}\left(a_{i k} \rightarrow^{\odot} r_{k}\right)=b_{i}$.

From Theorem 3.11, we obtain the following corollary.

Corollary 3.12. Let $U=\left\{u_{1}, \ldots, u_{n}\right\}$ be a set and $P \in L^{U \times U}$ a left $\odot$-preorder with $A_{i}(u)=P\left(u_{i}, u\right) \in L^{U}$. Then the following statements hold.

(1) $\bigvee_{j=1}^{n}\left(r_{j} \odot a_{i j}\right)=b_{i}$ is solvable iff $a_{i j}=\bigwedge_{k=1}^{n}\left(a_{k i} \rightarrow a_{k j}\right)=\bigwedge_{k=1}^{n}\left(a_{j k} \Rightarrow\right.$ $\left.a_{i k}\right) \leq b_{j} \Rightarrow b_{i}$ for all $i, j \in\{1, \ldots, n\}$.

(2) $\bigvee_{j=1}^{n}\left(a_{i j} \Rightarrow r_{j}\right)=b_{i}$ is solvable iff $a_{i j}=\bigwedge_{k=1}^{n}\left(a_{k i} \rightarrow a_{k j}\right)=\bigwedge_{k=1}^{n}\left(a_{j k} \Rightarrow\right.$ $\left.a_{i k}\right) \leq b_{i} \rightarrow b_{j}$ for all $i, j \in\{1, \ldots, n\}$.

Theorem 3.13. Let $A_{i} \in L^{U}$ be normal for $1 \leq i \leq n$ such that $a_{i i}=\top$. Then the following properties hold.

(1) $R^{\odot}=\left(R_{1}^{\odot}, \ldots, R_{n}^{\odot}\right)$ with $R_{j}^{\odot}=\bigvee_{i=1}^{n}\left(a_{i j} \odot b_{i}\right)$ is a solution of (5) iff $\bigvee_{k=1}^{n}\left(a_{i k} \odot a_{j k}\right) \leq\left(b_{j} \rightarrow b_{i}\right), \forall i, j \in\{1, \ldots, n\}$.

$(2){ }^{\odot} R=\left({ }^{\odot} R_{1}, \ldots,{ }^{\odot} R_{n}\right)$ with ${ }^{\odot} R_{k}=\bigvee_{i=1}^{n}\left(b_{i} \odot a_{i k}\right)$ is a solution of (6) iff $\bigvee_{k=1}\left(a_{j k} \odot a_{i k}\right) \leq\left(b_{j} \Rightarrow b_{i}\right), \forall i, j \in\{1, \ldots, n\}$. 
Proof. (1) Let $R^{\odot}=\left(R_{1}^{\odot}, \ldots, R_{n}^{\odot}\right)$ with $R_{j}^{\odot}=\bigvee_{i=1}^{n}\left(a_{i j} \odot b_{i}\right)$ be a solution of (5). Then

$$
\begin{aligned}
b_{i} & =\bigvee_{k=1}^{n}\left(a_{i k} \odot R_{k}^{\odot}\right)=\bigvee_{k=1}^{n}\left(a_{i k} \odot \bigvee_{i=1}^{n}\left(a_{i k} \odot b_{i}\right)\right) \\
& \geq a_{i k} \odot a_{j k} \odot b_{j}
\end{aligned}
$$

Hence $\bigvee_{k=1}^{n}\left(a_{i k} \odot a_{j k}\right) \leq\left(b_{j} \rightarrow b_{i}\right), \forall i, j$.

Conversely, since $A_{i} \in L^{U}$ is normal for $1 \leq i \leq n$, there exists $u_{i} \in U$ such that $a_{i i}=\top$. Hence $b_{i}=a_{i i} \odot a_{i i} \odot b_{i} \leq \bigvee_{k=1}^{n}\left(a_{i k} \odot R_{k}^{\odot}\right)$. Since $a_{i k} \odot a_{j k} \leq b_{j} \rightarrow b_{i}$ iff $a_{i k} \odot a_{j k} \odot b_{j} \leq b_{i}$, then $\bigvee_{k=1}^{n}\left(a_{i k} \odot R_{k}^{\odot}\right) \leq b_{i}$. So, $\bigvee_{k=1}^{n}\left(a_{i k} \odot R_{k}^{\odot}\right)=b_{i}$. Hence $R^{\odot}=\left(R_{1}^{\odot}, \ldots, R_{n}^{\odot}\right)$ with $R_{k}^{\odot}=\bigvee_{i=1}^{n}\left(a_{i k} \odot b_{i}\right)$ is a solution of $(5)$. Then

(2) Let $\odot R=\left(\odot R_{1}, \ldots, \odot R_{n}\right)$ with $\odot R_{k}=\bigvee_{i=1}^{n}\left(b_{i} \odot a_{i k}\right)$ is a solution of (6).

$$
\begin{aligned}
b_{i} & =\bigvee_{k=1}^{n}\left(\odot R_{k} \odot a_{i k}\right) \\
& =\bigvee_{k=1}^{n}\left(\bigvee_{j=1}^{n}\left(b_{j} \odot a_{j k}\right) \odot a_{i k}\right) \\
& \geq b_{j} \odot a_{j k} \odot a_{i k} .
\end{aligned}
$$

Hence $\bigvee_{k=1}^{n}\left(a_{i k} \odot a_{j k}\right) \leq b_{j} \Rightarrow b_{i}, \forall i, j \in\{1, \ldots, n\}$.

Conversely, since $A_{i} \in L^{U}$ is normal for $1 \leq i \leq n$, there exists $u_{i} \in U$ such that $a_{i i}=\top$. Hence $b_{i}=b_{i} \odot a_{i i} \odot a_{i i} \leq \bigvee_{k=1}^{n}\left(\odot R_{k} \circ a_{i k}\right)$. Since $a_{j k} \odot a_{i k} \leq b_{j} \Rightarrow$ $b_{i}$ iff $b_{j} \odot a_{j k} \odot a_{i k} \leq b_{i}$, then $\bigvee_{k=1}^{n}\left(\odot R_{k} \circ a_{i k}\right) \leq b_{i}$. So, $\bigvee_{k=1}^{n}\left(\odot R_{k} \circ a_{i k}\right)=b_{i}$. Hence ${ }^{\odot} R=\left({ }^{\odot} R_{1}, \ldots,{ }^{\odot} R_{n}\right)$ with ${ }^{\odot} R_{k}=\bigvee_{i=1}^{n}\left(b_{i} \odot a_{i k}\right)$ is a solution of (6).

Example 3.15.Let $K=\left\{(x, y) \in R^{2} \mid x>0\right\}$ be a set and we define an operation $\otimes: K \times K \rightarrow K$ as follows:

$$
\left(x_{1}, y_{1}\right) \otimes\left(x_{2}, y_{2}\right)=\left(x_{1} x_{2}, x_{1} y_{2}+y_{1}\right)
$$

Then $(K, \otimes)$ is a group with $e=(1,0),(x, y)^{-1}=\left(\frac{1}{x},-\frac{y}{x}\right)$.

For $\left(x_{1}, y_{1}\right),\left(x_{2}, y_{2}\right) \in K$, we define

$$
\begin{aligned}
& \left(x_{1}, y_{1}\right) \leq\left(x_{2}, y_{2}\right) \\
& \Leftrightarrow\left(x_{1}, y_{1}\right)^{-1} \odot\left(x_{2}, y_{2}\right) \in P, \\
& \left(x_{2}, y_{2}\right) \odot\left(x_{1}, y_{1}\right)^{-1} \in P \\
& \Leftrightarrow x_{1}<x_{2} \text { or } x_{1}=x_{2}, y_{1} \leq y_{2} .
\end{aligned}
$$

Then $(K, \leq \otimes)$ is a lattice-group. (ref. [1])

The structure $\left(L, \odot, \Rightarrow, \rightarrow,\left(\frac{1}{2}, 1\right),(1,0)\right)$ is a generalized residuated lattice where $\perp=\left(\frac{1}{2}, 1\right)$ is the least element and $T=(1,0)$ is the greatest element 
from the following statements:

$$
\begin{aligned}
\left(x_{1}, y_{1}\right) \odot\left(x_{2}, y_{2}\right) & =\left(x_{1}, y_{1}\right) \otimes\left(x_{2}, y_{2}\right) \vee\left(\frac{1}{2}, 1\right) \\
& =\left(x_{1} x_{2}, x_{1} y_{2}+y_{1}\right) \vee\left(\frac{1}{2}, 1\right), \\
\left(x_{1}, y_{1}\right) \Rightarrow\left(x_{2}, y_{2}\right) & =\left(\left(x_{1}, y_{1}\right)^{-1} \otimes\left(x_{2}, y_{2}\right)\right) \wedge(1,0) \\
& =\left(\frac{x_{2}}{x_{1}}, \frac{y_{2}-y_{1}}{x_{1}}\right) \wedge(1,0), \\
\left(x_{1}, y_{1}\right) \rightarrow\left(x_{2}, y_{2}\right) & =\left(\left(x_{2}, y_{2}\right) \otimes\left(x_{1}, y_{1}\right)^{-1}\right) \wedge(1,0) \\
& =\left(\frac{x_{2}}{x_{1}},-\frac{x_{2} y_{1}}{x_{1}}+y_{2}\right) \wedge(1,0) .
\end{aligned}
$$

Let $U=\left\{u_{1}, u_{2}, u_{3}\right\}$ be a set. Define $P \in L^{U \times U}$ as

$$
P=\left(\begin{array}{ccc}
(1,0) & \left(\frac{5}{8}, \frac{5}{2}\right) & \left(\frac{5}{6}, \frac{5}{3}\right) \\
\left(\frac{5}{7}, \frac{30}{7}\right) & (1,0) & \left(\frac{5}{8},-\frac{5}{4}\right) \\
(1,-2) & \left(\frac{5}{7}, \frac{10}{3}\right) & (1,0)
\end{array}\right)
$$

Since $P \circ P \leq P$ and $P^{t} \circ P^{t} \leq P^{t}$, by Remark 3.5(2), we easily show that $P$ is a right $\odot$-preorder and $P^{t}$ is a left $\odot$-preorder.

(1) Put $A_{i}=\left(A_{i}\left(u_{1}\right), A_{i}\left(u_{2}\right), A_{i}\left(u_{3}\right)\right)$ for each $i=\{1,2,3\}$ as follows

$$
\begin{gathered}
A_{1}=\left((1,0),\left(\frac{5}{8}, \frac{5}{2}\right),\left(\frac{5}{6}, \frac{5}{3}\right)\right) \\
A_{2}=\left(\left(\frac{5}{7}, \frac{30}{7}\right),(1,0),\left(\frac{5}{8},-\frac{5}{4}\right)\right) \\
A_{3}=\left((1,-2),\left(\frac{5}{7}, \frac{10}{3}\right),(1,0)\right) \\
b=\left(b_{1}, b_{2}, b_{3}\right)=\left(\left(\frac{3}{5}, 1\right),\left(\frac{5}{6},-3\right),\left(\frac{2}{3}, 2\right)\right)
\end{gathered}
$$

$$
\begin{aligned}
& (1,0) \odot r_{1} \vee\left(\frac{5}{8}, \frac{5}{2}\right) \odot r_{2} \vee\left(\frac{5}{6}, \frac{5}{3}\right) \odot r_{3}=\left(\frac{3}{5}, 1\right) \\
& \left(\frac{5}{7}, \frac{30}{7}\right) \odot r_{1} \vee(1,0) \odot r_{2} \vee\left(\frac{5}{8},-\frac{5}{4}\right) \odot r_{3}=\left(\frac{5}{6},-3\right) \\
& (1,-2) \odot r_{1} \vee\left(\frac{5}{7}, \frac{10}{3}\right) \odot r_{2} \vee(1,0) \odot r_{3}=\left(\frac{2}{3}, 2\right)
\end{aligned}
$$

Since $P=\left(a_{i j}\right)$ is a right $\odot$-preorder, by Corollary 3.8(1), $A_{i}(u)=P\left(u_{i}, u\right)$ and $A_{i}\left(u_{j}\right)=\bigwedge_{u \in U}\left(A_{j}(u) \rightarrow A_{i}(u)\right) ;$;.e. $a_{i j}=\bigwedge_{k=1}^{3}\left(a_{j k} \rightarrow a_{i k}\right)$. Since $a_{i j} \leq b_{j} \rightarrow$ $b_{i}$ for all $i, j=\{1,2,3\}$, by Corollary $3.10(1), \bigvee_{j=1}^{3}\left(a_{i j} \odot r_{j}\right)=b_{i}$ is solvable. By Theorem 3.2, $r \Rightarrow=\left(\left(\frac{3}{5}, 1\right),\left(\frac{5}{6},-3\right),\left(\frac{2}{3}, 2\right)\right)$ with $r_{j} \Rightarrow \bigwedge_{k=1}^{3}\left(a_{k j} \Rightarrow b_{k}\right)$ is a solution.

Since $(1,-2)=\bigvee_{j=1}^{3}\left(a_{1 j} \odot a_{3 j}\right) \not \leq\left(b_{3} \rightarrow b_{1}\right)=\left(\frac{9}{10},-\frac{4}{5}\right)$, by Theorem 3.13 (1), $R^{\odot}=\left(\left(\frac{2}{3}, 0\right),\left(\frac{5}{6},-3\right),\left(\frac{2}{3}, 2\right)\right)$ with $R_{k}^{\odot}=\bigvee_{i=1}^{n}\left(a_{i k} \odot b_{i}\right)$ is not a solution of (9). 


$$
\begin{aligned}
& r_{1} \odot(1,0) \vee r_{2} \odot\left(\frac{5}{8}, \frac{5}{2}\right) \vee r_{3} \odot\left(\frac{5}{6}, \frac{5}{3}\right)=\left(\frac{3}{5}, 1\right)\left(\frac{2}{3}, 2\right) \\
& r_{1} \odot\left(\frac{5}{7}, \frac{30}{7}\right) \vee r_{2} \odot(1,0) \vee r_{3} \odot\left(\frac{5}{8},-\frac{5}{4}\right)=\left(\frac{5}{6},-3\right) \\
& r_{1} \odot(1,-2) \vee r_{2} \odot\left(\frac{5}{7}, \frac{10}{3}\right) \vee r_{3} \odot(1,0)=\left(\frac{2}{3}, 2\right)
\end{aligned}
$$

Since $P=\left(a_{i j}\right)$ is a left $\odot$-preorder, by Corollary 3.8(2), $A_{i}(u)=P\left(u_{i}, u\right)$ and $A_{i}\left(u_{j}\right)=\bigwedge_{u \in U}\left(A_{j}(u) \Rightarrow A_{i}(u)\right)$;i.e. $a_{i j}=\bigwedge_{k=1}^{3}\left(a_{j k} \Rightarrow a_{i k}\right)$. Since $a_{i j} \leq$ $b_{j} \Rightarrow b_{i}$ for all $i, j=\{1,2,3\}$, by Corollary $3.12(1), \bigvee_{k=1}^{3}\left(r_{k} \odot a_{i k}\right)=b_{i}$ is solvable. Then $r \rightarrow=\left(\left(\frac{3}{5}, 1\right),\left(\frac{5}{6},-3\right),\left(\frac{2}{3}, 2\right)\right)$ with $r_{k} \rightarrow=\bigwedge_{j=1}^{3}\left(a_{j k} \rightarrow b_{j}\right)$ is a solution of $(10)$. Since $(1,-2)=\bigvee_{j=1}^{3}\left(a_{3 j} \odot a_{1 j}\right) \not \leq\left(b_{3} \Rightarrow b_{1}\right)=\left(\frac{9}{10},-\frac{3}{2}\right)$, by Theorem 3.13(2), ${ }^{\odot} R=\left(\left(\frac{2}{3}, \frac{2}{3}\right),\left(\frac{5}{6},-3\right),\left(\frac{2}{3}, 2\right)\right)$ with ${ }^{\odot} R_{k}=\bigvee_{i=1}^{3}\left(b_{i} \odot a_{i k}\right)$ is not a solution of (10).

$(1-3)$

$$
\begin{aligned}
& (1,0) \rightarrow r_{1} \wedge\left(\frac{5}{8}, \frac{5}{2}\right) \rightarrow r_{2} \wedge\left(\frac{5}{6}, \frac{5}{3}\right) \rightarrow r_{3}=\left(\frac{3}{5}, 1\right) \\
& \left(\frac{5}{7}, \frac{30}{7}\right) \rightarrow r_{1} \wedge(1,0) \rightarrow r_{2} \wedge\left(\frac{5}{8},-\frac{5}{4}\right) \rightarrow r_{3}=\left(\frac{5}{6},-3\right) \\
& (1,-2) \rightarrow r_{1} \wedge\left(\frac{5}{7}, \frac{10}{3}\right) \rightarrow r_{2} \wedge(1,0) \rightarrow r_{3}=\left(\frac{2}{3}, 2\right)
\end{aligned}
$$

Since $P=\left(a_{i j}\right)$ is a right $\odot$-preorder, by Corollary 3.8(1), $A_{i}(u)=P\left(u_{i}, u\right)$ and $A_{i}\left(u_{j}\right)=\bigwedge_{u \in U}\left(A_{j}(u) \rightarrow A_{i}(u)\right)$;i.e. $a_{i j}=\bigwedge_{k=1}^{3}\left(a_{j k} \rightarrow a_{i k}\right)$. Since $(1,-2)=$ $a_{31} \not \leq b_{1} \Rightarrow b_{3}=\left(\frac{9}{10},-\frac{3}{2}\right)$, by corollary $3.10(2), \bigwedge_{k=1}^{3}\left(a_{i k} \rightarrow r_{k}\right)=b_{i}$ is not solvable. Thus $\odot r=\left(\left(\frac{2}{3},-\frac{4}{3}\right),\left(\frac{5}{6},-3\right),\left(\frac{2}{3}, 0\right)\right)$ with $\odot_{r_{k}}=\bigvee_{j=1}^{n}\left(b_{j} \odot a_{j k}\right)$ is not a solution of (11).

$$
\begin{aligned}
& (1,0) \Rightarrow r_{1} \wedge\left(\frac{5}{8}, \frac{5}{2}\right) \Rightarrow r_{2} \wedge\left(\frac{5}{6}, \frac{5}{3}\right) \Rightarrow r_{3}=\left(\frac{3}{5}, 1\right) \\
& \left(\frac{5}{7}, \frac{30}{7}\right) \Rightarrow r_{1} \wedge(1,0) \Rightarrow r_{2} \wedge\left(\frac{5}{8},-\frac{5}{4}\right) \Rightarrow r_{3}=\left(\frac{5}{6},-3\right) \\
& (1,-2) \Rightarrow r_{1} \wedge\left(\frac{5}{7}, \frac{10}{3}\right) \Rightarrow r_{2} \wedge(1,0) \Rightarrow r_{3}=\left(\frac{2}{3}, 2\right)
\end{aligned}
$$

Since $P=\left(a_{i j}\right)$ is a left $\odot$-preorder, by Corollary 3.8(1), $A_{i}(u)=P\left(u_{i}, u\right)$ and $A_{i}\left(u_{j}\right)=\bigwedge_{u \in U}\left(A_{j}(u) \Rightarrow A_{i}(u)\right)$;i.e. $a_{i j}=\bigwedge_{k=1}^{3}\left(a_{j k} \Rightarrow a_{i k}\right)$. Since $(1,-2)=$ $a_{31} \not \leq b_{1} \rightarrow b_{3}=\left(\frac{9}{10},-\frac{4}{5}\right)$, by Corollary $3.12(2), \bigwedge_{k=1}^{3}\left(a_{i k} \Rightarrow r_{k}\right)=b_{i}$ is not solvable. Thus $\odot_{r}=\left(\left(\frac{2}{3},-\frac{4}{3}\right),\left(\frac{5}{6},-3\right),\left(\frac{2}{3}, 0\right)\right)$ with $\odot_{r_{k}}=\bigvee_{j=1}^{n}\left(a_{j k} \odot b_{j}\right)$ is not a solution of (12).

(2) Put $A_{i} \in L^{U}$ for $i \in\{1,2,3\}$ as same in (1) and

$$
b=\left(b_{1}, b_{2}, b_{3}\right)=\left(\left(\frac{2}{3}, 2\right),\left(\frac{5}{6},-3\right),\left(\frac{3}{5}, 1\right)\right) .
$$




$$
\begin{aligned}
& (1,0) \rightarrow r_{1} \wedge\left(\frac{5}{8}, \frac{5}{2}\right) \rightarrow r_{2} \wedge\left(\frac{5}{6}, \frac{5}{3}\right) \rightarrow r_{3}=\left(\frac{2}{3}, 2\right) \\
& \left(\frac{5}{7}, \frac{30}{7}\right) \rightarrow r_{1} \wedge(1,0) \rightarrow r_{2} \wedge\left(\frac{5}{8},-\frac{5}{4}\right) \rightarrow r_{3}=\left(\frac{5}{6},-3\right) \\
& (1,-2) \rightarrow r_{1} \wedge\left(\frac{5}{7}, \frac{10}{3}\right) \rightarrow r_{2} \wedge(1,0) \rightarrow r_{3}=\left(\frac{3}{5}, 1\right)
\end{aligned}
$$

Since $P=\left(a_{i j}\right)$ is a right $\odot$-preorder, by Corollary 3.8(1), $A_{i}(u)=P\left(u_{i}, u\right)$ and $A_{i}\left(u_{j}\right)=\bigwedge_{u \in U}\left(A_{j}(u) \rightarrow A_{i}(u)\right)$;i.e. $a_{i j}=\bigwedge_{k=1}^{3}\left(a_{j k} \rightarrow a_{i k}\right)$. Since $a_{i j} \leq b_{i} \Rightarrow$ $b_{j}$, by Corollary 3.10(2), $\bigwedge_{k=1}^{3}\left(a_{i k} \rightarrow r_{k}\right)=b_{i}$ is solvable. By Theorem 3.2(4). $\odot_{r}=\left(\left(\frac{2}{3}, 2\right),\left(\frac{5}{6},-3\right),\left(\frac{3}{5}, 1\right)\right)$ with ${ }^{\odot} r_{k}=\bigvee_{j=1}^{3}\left(b_{j} \odot a_{j k}\right)$ is a solution of $(13)$.

$$
\begin{aligned}
& (1,0) \Rightarrow r_{1} \wedge\left(\frac{5}{8}, \frac{5}{2}\right) \Rightarrow r_{2} \wedge\left(\frac{5}{6}, \frac{5}{3}\right) \Rightarrow r_{3}=\left(\frac{2}{3}, 2\right) \\
& \left(\frac{5}{7}, \frac{30}{7}\right) \Rightarrow r_{1} \wedge(1,0) \Rightarrow r_{2} \wedge\left(\frac{5}{8},-\frac{5}{4}\right) \Rightarrow r_{3}=\left(\frac{5}{6},-3\right) \\
& (1,-2) \Rightarrow r_{1} \wedge\left(\frac{5}{7}, \frac{10}{3}\right) \Rightarrow r_{2} \wedge(1,0) \Rightarrow r_{3}=\left(\frac{3}{5}, 1\right)
\end{aligned}
$$

Since $P=\left(a_{i j}\right)$ is a left $\odot$-preorder, by Corollary 3.8(2), $A_{i}(u)=P\left(u_{i}, u\right)$ and $A_{i}\left(u_{j}\right)=\bigwedge_{u \in U}\left(A_{j}(u) \Rightarrow A_{i}(u)\right)$;i.e. $a_{i j}=\bigwedge_{k=1}^{3}\left(a_{j k} \Rightarrow a_{i k}\right)$. Since $a_{i j} \leq b_{i} \rightarrow$ $b_{j}$, by Corollary 3.12(2), $\bigwedge_{k=1}^{3}\left(a_{i k} \Rightarrow r_{k}\right)=b_{i}$ is solvable. By Theorem 3.2(3), $\odot_{r}=\left(\left(\frac{2}{3}, 2\right),\left(\frac{5}{6},-3\right),\left(\frac{3}{5}, 1\right)\right)$ with $r_{k}^{\odot}=\bigvee_{j=1}^{3}\left(a_{j k} \odot b_{j}\right)$ is a solution of (14).

$$
\begin{aligned}
& (1,0) \odot r_{1} \vee\left(\frac{5}{8}, \frac{5}{2}\right) \odot r_{2} \vee\left(\frac{5}{6}, \frac{5}{3}\right) \odot r_{3}=\left(\frac{2}{3}, 2\right) \\
& \left(\frac{5}{7}, \frac{30}{7}\right) \odot r_{1} \vee(1,0) \odot r_{2} \vee\left(\frac{5}{8},-\frac{5}{4}\right) \odot r_{3}=\left(\frac{5}{6},-3\right) \\
& (1,-2) \odot r_{1} \vee\left(\frac{5}{7}, \frac{10}{3}\right) \odot r_{2} \vee(1,0) \odot r_{3}=\left(\frac{3}{5}, 1\right)
\end{aligned}
$$

Since $(1,-2)=a_{31} \not \leq b_{1} \rightarrow b_{3}=\left(\frac{9}{10},-\frac{4}{5}\right)$, by Corollary 3.10(1), $\bigvee_{k=1}^{3}\left(a_{i k} \odot\right.$ $\left.r_{k}\right)=b_{i}$ is not solvable. Then $r_{k}^{\Rightarrow}=\bigwedge_{j=1}^{3}\left(a_{j k} \Rightarrow b_{j}\right)$ is not a solution such that $r \Rightarrow=\left(\left(\frac{3}{5}, 3\right),\left(\frac{5}{6},-3\right),\left(\frac{2}{3}, 2\right)\right)$..

$$
\begin{aligned}
& r_{1} \odot(1,0) \vee r_{2} \odot\left(\frac{5}{8}, \frac{5}{2}\right) \vee r_{3} \odot\left(\frac{5}{6}, \frac{5}{3}\right)=\left(\frac{2}{3}, 2\right) \\
& r_{1} \odot\left(\frac{5}{7}, \frac{30}{7}\right) \vee r_{2} \odot(1,0) \vee r_{3} \odot\left(\frac{5}{8},-\frac{5}{4}\right)=\left(\frac{5}{6},-3\right) \\
& r_{1} \odot(1,-2) \vee r_{2} \odot\left(\frac{5}{7}, \frac{10}{3}\right) \vee r_{3} \odot(1,0)=\left(\frac{3}{5}, 1\right)
\end{aligned}
$$

Since $(1,-2)=a_{31} \not \leq b_{1} \Rightarrow b_{3}=\left(\frac{9}{10},-\frac{3}{2}\right)$, by Corollary $3.12(1), \bigvee_{k=1}^{3}\left(r_{k} \odot\right.$ $\left.a_{i k}\right)=b_{i}$ is not solvable. Then $r \rightarrow=\left(\left(\frac{3}{5}, \frac{11}{5}\right),\left(\frac{5}{6},-3\right),\left(\frac{2}{3}, 2\right)\right)$ with $r_{k} \rightarrow=$ $\bigwedge_{j=1}^{3}\left(a_{j k} \rightarrow b_{j}\right)$ is not a solution of (16).

(3) Put $A_{i}=\left(A_{i}\left(u_{1}\right), A_{i}\left(u_{2}\right), A_{i}\left(u_{3}\right)\right)$ for each $i=\{1,2,3\}$ as follows

$$
A_{1}=\left((1,0),\left(\frac{5}{8}, \frac{5}{2}\right),\left(\frac{3}{5}, \frac{5}{3}\right)\right)
$$




$$
\begin{gathered}
A_{2}=\left(\left(\frac{5}{7}, \frac{30}{7}\right),(1,0),\left(\frac{5}{8},-\frac{5}{4}\right)\right) \\
A_{3}=\left(\left(\frac{2}{3},-2\right),\left(\frac{5}{7}, \frac{10}{3}\right),(1,0)\right) \\
b=\left(b_{1}, b_{2}, b_{3}\right)=\left(\left(\frac{5}{6},-3\right),\left(\frac{3}{4}, 1\right),(1,-2)\right)
\end{gathered}
$$

$$
\begin{aligned}
& (1,0) \odot r_{1} \vee\left(\frac{5}{8}, \frac{5}{2}\right) \odot r_{2} \vee\left(\frac{3}{5}, \frac{5}{3}\right) \odot r_{3}=\left(\frac{5}{6},-3\right) \\
& \left(\frac{5}{7}, \frac{30}{7}\right) \odot r_{1} \vee(1,0) \odot r_{2} \vee\left(\frac{5}{8},-\frac{5}{4}\right) \odot r_{3}=\left(\frac{3}{4}, 1\right) \\
& \left(\frac{2}{3},-2\right) \odot r_{1} \vee\left(\frac{5}{7}, \frac{10}{3}\right) \odot r_{2} \vee(1,0) \odot r_{3}=(1,-2)
\end{aligned}
$$

Put $P=\left(a_{i j}\right)$. Since $P \circ P \leq P$ and $P^{t} \circ P^{t} \leq P^{t}$, by Remark 3.5(2), $P$ is a right $\odot$-preorder and $P$ is a left $\odot$-preorder. Since $a_{i j} \leq b_{j} \rightarrow b_{i}$ for all $i, j=\{1,2,3\}$, by Corollary $3.10(1), \bigvee_{k=1}^{3}\left(a_{i k} \odot r_{k}\right)=b_{i}$ is solvable. From Theorem 3.2(1), $r \Rightarrow=\left(\left(\frac{5}{6},-3\right),\left(\frac{3}{4}, 1\right),(1,-2)\right)$ with $r_{k}^{\Rightarrow}=\bigwedge_{j=1}^{3}\left(a_{j k} \Rightarrow b_{j}\right)$ is a solution.

Since $\bigvee_{k=1}^{3}\left(a_{i k} \odot a_{j k}\right) \leq\left(b_{j} \rightarrow b_{i}\right)$, by Theorem $3.13(1), R^{\odot}=\left(\left(\frac{5}{6},-3\right)\right.$, $\left.\left(\frac{3}{4}, 1\right),(1,-2)\right)$ with $R_{k}^{\odot}=\bigvee_{i=1}^{3}\left(a_{i k} \odot b_{i}\right)$ is a solution of $(17)$.

$$
\begin{aligned}
& r_{1} \odot(1,0) \vee r_{2} \odot\left(\frac{5}{8}, \frac{5}{2}\right) \vee r_{3} \odot\left(\frac{3}{5}, \frac{5}{3}\right)=\left(\frac{5}{6},-3\right) \\
& r_{1} \odot\left(\frac{5}{7}, \frac{30}{7}\right) \vee r_{2} \odot(1,0) \vee r_{3} \odot\left(\frac{5}{8},-\frac{5}{4}\right)=\left(\frac{3}{4}, 1\right) \\
& r_{1} \odot\left(\frac{2}{3},-2\right) \vee r_{2} \odot\left(\frac{5}{7}, \frac{10}{3}\right) \vee r_{3} \odot(1,0)=(1,-2)
\end{aligned}
$$

Since $a_{i j} \leq b_{j} \Rightarrow b_{i}$ for all $i, j=\{1,2,3\}$, by Corollary $3.10(2), \bigvee_{k=1}^{3}\left(r_{k} \odot\right.$ $\left.a_{i k}\right)=b_{i}$ is solvable. Then $r^{\rightarrow}=\left(\left(\frac{5}{6},-3\right),\left(\frac{3}{4}, 1\right),(1,-2)\right)$ with $r_{k}=\bigwedge_{j=1}^{3}\left(a_{j k} \rightarrow\right.$ $\left.b_{j}\right)$ is a solution. Since $\bigvee_{k=1}^{3}\left(a_{i k} \odot a_{j k}\right) \leq\left(b_{i} \Rightarrow b_{j}\right)$, by Theorem $3.13(2)$, ${ }^{\circ} R=\left(\left(\frac{5}{6},-3\right),\left(\frac{3}{4}, 1\right),(1,-2)\right)$ with $\odot R_{k}=\bigvee_{i=1}^{n}\left(b_{i} \odot a_{i k}\right)$ is a solution of $(18)$.

\section{References}

[1] G. Birkhoff, Lattice Theory, Amer. Math. Soc. Colloq. Publ., 25, New York, 1968.

[2] A. Dvurecenskij, Pseudo MV-algebras are intervals in l-groups, J. Australian Math. Soc., 72 (2002), 427-445, doi: 10.1017/s1446788700036806. 
[3] A. Dvurecenskij, On pseudo MV-algebras, Soft Computing,5 (2001), 347354, doi: $10.1007 / \mathrm{s} 005000100136$.

[4] N. Galatos, C. Tsinakis, Generalized MV-alebras, J. of Algebra, 283 (2005), 254-291, doi: 10.1016/j. jalgebra,2004,07,002.

[5] G. Georgescu, A. lorgulescu, Pseudo MV-algebras, Multiple-Valued Logics, 6 (2001), 193-215.

[6] G. Georgescu, A. Popescu, Non-commutative fuzzy Galois connections, Soft Computing, 7 (2003), 458-467.

[7] G. Georgescu, A. Popescu, Non-commutative fuzzy structures and pairs of weak negations, Fuzzy Sets and Systems, 143 (2004), 129-155, doi: $10.1016 /$ j. fss, $2003,06,004$.

[8] S. Gottwald, On the existence of soltion of systems of fuzzy equations, Fuzzy Sets and Systems, 12 (1984), 301-302, doi: 10.1016/01650114(84)90076-9.

[9] A.A. Molai, E. Khorram, An algorithm for solving fuzzy relation equations with max-T composition operator, Information Sciences , 178 (2008), 1293-1308, doi: 10.1016/j. ins,2007,10,010.

[10] W. Pedrycz, Inverse problem in fuzzy relation equations, Fuzzy Sets and Systems, 36 (1990), 277-291, doi: 10.1016/0165-0114(90)90185-9.

[11] I. Perfilieva, Fuzzy function as an approximate solution to a system of fuzzy relation equations, Fuzzy Sets and Systems, 147 (2004), 363-383, doi: 10.1016/0165-0114(90)90185-9.

[12] I. Perfilieva, L. Noskova, System of fuzzy relation equations with inf- composition: Commplete set of solutions, Fuzzy Sets and Systems, 159 (2008), 2256-2271, doi: 10.1016/j. fss,2007,12,012.

[13] I. Perfilieva, Finitary solvablity conditions for system of fuzzy relation equations Information Sciences, 234 (2013), 29-43, doi: 10.1016/j. ins, $2011,04,035$.

[14] E. Sanchez, Resolution of composite fuzzy relation equations, Inform. and Control, 30 (1976), 38-48, doi: 10.1016/s0019-9958(76)90446-0. 
[15] B.S. Shieh, Solutions of fuzzy relation equations based on continuous t-norms, Information Sciences, 177 (2007), 4208-4215, doi: 10.1016/j. ins, 2007,04,006. 